\title{
Prospects for fifth force fade
}

\section{Conflicting observations of the gravitational-like interactions which have engendered talk about the fifth force might have been reconciled if it were not for another set of data.}

Whatever has happened to the fifth force? Eighteen months have passed since the existence of a non-newtonian interaction between massive objects was postulated by Fischbach, Sudarsky, Szafer, Talmadge and Aronson on the basis of their reanalysis of the measurements by Eötvos, in the $1920 \mathrm{~s}$, of the apparent 'gravitational' interaction between objects of various composition.

That the old data are shot through with anomalies is not surprising. What Fischbach et al. say is that the anomalies could be removed if there is an extra interaction between material objects whose strength is determined not by the mass of an object but by its content of baryons (neutrons and/or protons) which, because of nuclear packing fractions and non-zero electronic masses, is not related to the total mass of objects in the same way for all materials.

At the outset, the advent of the fifth force was both welcome and disconcerting. On the face of things, it has the advantage of being able to account for the way in which geophysical measurements of the gravitational constant, where one of the sources of gravitational attraction is an object such as the Earth, tend to be consistently higher than those derived from laboratory measurements (see Nature 319, 173; 1986). Among theorists, the development was also welcome on general grounds of completeness: baryon number, like net electrical charge, is conserved in the standard particle theories, but appears not to play a part in the determination of an interaction between objects. The bad news was merely that a non-newtonian interaction (with a short range somewhere between a few metres and $10 \mathrm{~km}$ ) would have been an unwelcome, if intriguing, complication of an orderly world.

Either way, it has been clear that the issue will be settled only by experiment, which explains why so much ingenuity has been lavished on novel measurements in the past 18 months. Simply repeating the Eötvos measurements has naturally not been appealing. Given that the supposed force is very small compared with authentic gravitation, the best hope of detecting it rests on measurements of apparently gravitational attractions in circumstances where there is a great bulk of matter generating fifth force in some recognizable direction.

One of the most ingenious schemes (due to Professor S.K.Runcorn of the
University of Newcastle upon Tyne) would entail the measurement of gravitational acceleration near the energy storage plant at Dinorwic in North Wales; gravimeters near the site should behave differently at different distances from the reservoir filled with water at off-peak times in the demand for electricity in Britain. Others have been busy off the faces of cliffs or beneath hillsides that spring out of an otherwise uniform topogrophy. Unfortunately for the fifth force, the results so far have been mixed.

Indeed, the two experiments so far described have given flatly contradictory results. Thus $\mathrm{P}$. Thieberger, working on an observation platform on the cliff-face of the Palisades, found a horizontal force between the cliff-face and a copper sphere floating in a tank of water (Phys. Rev. Lett. 58, 1066; 1987). On the other hand, a group from the University of Washington (C.W.Stubbs et al. Phys. Rev. Lett. 58, $1070 ; 1987)$ reported measurements with a copper and beryllium torsion balance installed on a hillside on the Seattle campus which flatly contradicted Thieberger's result or which, more accurately, fixed an upper limit for the magnitude of the supposed fifth force at two orders of magnitude less than that inferred from the reanalysis of the Eötvos measurements.

What, in such circumstances, should experimentalists do? The standard procedure is to pick over the other set of data, looking for sources of systematic error that might account for the discrepancy, a process that can be quite acrimonious. On this occasion, the Seattle group, almost as if it regretted having helped to make an interesting problem go away, has followed an ingenious tack by asking "What if we are both right?" (see Adelberger, E.G. et al. Phys. Rev. Lett. 59, 849; 1987).

How could that be? The suggestion is that the 'charge' responsible for the fifth force is not simply the baryon number of an interacting object but some combination of baryon number, $B$, and lepton number, $L$, conveniently (and without loss of generality) written as $q=B \cos \theta+$ $L \sin \theta$. What this implies is that the magnitude of a fifth-force attraction will be determined by the ratio of baryons to leptons in the test material. Given two accurate measurements, at Seattle and New York, it is plainly possible to find some value of the angle $\theta$ that will reconcile the null result at Seattle with the positive result off the Palisades cliffside. For what it is worth, $\theta$ comes out at $-11^{\circ}$.

So are the two apparently conflicting measurements merely manifestations of the same underlying phenomenon? The snag is that, while it may be possible to fit a sine curve to two arbitrary points, the same fit can also be used to define a more sensitive test of how good the fit may be. Adelberger et al. now simply figure out from their sine curve that, if the copper and copper/beryllium results can be reconciled at $\theta=-11^{\circ}$, replacing the copper component of their torsion balance by aluminium should give a positive result under the same condition, although not as large as that of Thieberger.

The result is again a disappointment for believers in the fifth force. The torsion balance constructed from beryllium and aluminium spheres (two of each, arranged asymmetrically to form a fifth-force charge dipole) gives a null result, or one smaller by two orders of magnitude than required to account for Thieberger's result.

Where the argument will go from here is a matter of conjecture, although the stimulus engendered by excitement about the fifth force for accurate measurements of gravitational acceleration $(g)$ under widely different circumstances may indeed soon lead to a better understanding of why the gravitational constant $(G)$, which fixes the magnitude of the newtonian gravitational force, is still among the least well defined of the natural constants.

If, by good luck, a much more accurate value of $G$ should emerge, we should all be the gainers. On the theoretical side, the case for believing that baryon and lepton numbers should have roles somehow analogous to electric charge, while only a formality in most arguments so far, should not be too lightly dismissed. Why not?

The obvious difficulty is that, if these numbers separately or jointly are to function as charges acting as sources for an interaction between material objects, the force will have to be mediated by particles of some kind. The snag here is that the particle called the axion, the existence of which is predicated by the strict application of parity and time reversal in weak interactions, and which has been canvassed both as a mediator of the nonnewtonian gravitational force and as the constituent of 'dark' intragalactic matter, has been having a bad time recently in attempts to demonstrate its experimental reality. 